\title{
La gestión del conocimiento y su aporte a la competitividad en las organizaciones: revisión sistemática de literatura ${ }^{*}$
}

\section{Knowledge management and its contribution to competitiveness in organizations: systematic review of literature}

\section{A gestão do conhecimento e sua contribuição para a competitividade nas organizações: revisão sistemática de literatura}

Mara Rocí Benavides Reina"

Asesora independiente

Ximena Lucía Pedraza-Najar"*

Universidad Santo Tomás en convenio con Icontec

\section{RESUMEN}

La dinámica global de los mercados ha generado necesidades específicas para que las organizaciones sean sostenibles en el tiempo, puesto que la turbulencia y el cambio son permanentes. Este escenario ha permitido identificar que no solo la actualización tecnológica se convierte en un reto para la estrategia organizacional, sino que el valor que tiene el conocimiento es insuperable y que su direccionamiento y control son y serán

Artículo de revisión. Citar como: Benavides, M., Pedraza, X. (2018). La gestión del conocimiento y su aporte a la competitividad en las organizaciones: revisión sistemática de literatura. SIGNOS, 10(2), 175-191. DOI: https://doi.org/10.15332/s2145-1389.2018.0002.10

** Magíster en Calidad y Gestión Integral, especialista en Docencia Universitaria, licenciada en Biología. Asesora independiente. Correo electrónico: benavidesreina@gmail.com

*** Doctora en Administración, magíster en Calidad y Gestión Integral, especialista en Gestión de la Producción, la Calidad y la Tecnología, especialista en Gerencia de Procesos, Calidad e Innovación. Microbióloga. Docente de la Maestría en Calidad y Gestión Integral. Universidad Santo Tomás. Correo electrónico: ximenapedraza@yahoo.com 
prioridad en todas las organizaciones de todos los sectores productivos y de servicios, especialmente en las pymes, quienes aportan a la economía global de manera significativa. Por esta razón, la presente revisión sistemática de literatura de los últimos 10 años, tiene como objetivo determinar la incursión de la gestión del conocimiento como ventaja competitiva que asegure la subsistencia organizacional en un entorno interno y externo cambiante. Se identifica la importancia y acogida que ha tenido en las pymes y en sectores específicos de la economía, como son: el petrolero, el educativo (educación superior), el solidario (tercer sector), el manufacturero y el bancario, a partir de los cuales, se lograron determinar las dimensiones en las que se enfatiza la gestión del conocimiento.

Palabras clave: gestión del conocimiento, competitividad, pymes, innovación, capital intelectual.

\section{ABSTRACT}

The global dynamics of markets has generated specific needs for organizations to be sustainable over time, since turbulence and change are permanent. This scenario has made possible to identify that not only technological update becomes a challenge for the organizational strategy, but the value knowledge has is insurmountable and its direction and control are and will be a priority in all the organizations of all the productive and services sectors, especially in SMEs, who contribute significantly to the global economy. For this reason, this systematic review of literature, of the last 10 years, aims to determine the incursion of knowledge management as a competitive advantage that ensures organizational subsistence in a changing internal and external environment. It identifies the importance and reception the
SMEs have had and in specific sectors of the economy, such as: oil, education (higher education), solidarity (third sector), manufacturing and banking, from which, it was possible to determine the dimensions in which knowledge management is emphasized.

Keywords: knowledge management, competitiveness, SMEs, innovation, intellectual capital.

\section{RESUMO}

A dinâmica global dos mercados gerou necessidades específicas para que as organizações sejam sustentáveis no tempo, uma vez que a turbulência e a mudança são permanentes. Este cenário permitiu identificar que não só a atualização tecnológica se torna em um desafio para a estratégia organizacional, também 0 valor que tem o conhecimento é insuperável e que seu direcionamento e controle são e serão uma prioridade em todas as organizações de todos os setores produtivos e de serviços, especialmente nas PME, que contribuem significativamente para a economia global. Por essa razão, a presente revisão sistemática da literatura, dos últimos 10 anos, tem como objetivo determinar a incursão da gestão do conhecimento como vantagem competitiva que garante a subsistência organizacional em um ambiente interno e externo em mudança. Identifica a importância e a recepção que teve nas PMEs e em setores específicos da economia, tais como: petróleo, educação (ensino superior), solidário (terceiro setor), manufatura e bancário, a partir dos quais foi possível determinar as dimensões nas quais a gestão do conhecimento é enfatizada.

Palavras-chave: gestão do conhecimento, competitividade, PMEs, inovação, capital intelectual. 


\section{INTRODUCCIÓN}

El inicio de la era del conocimiento se presenta a finales de los años noventa como respuesta de las organizaciones a los cambios sociales, económicos y tecnológicos caracterizados por los desarrollos tecnológicos, la aparición de las tecnologías de la información y la comunicación, la creciente importancia del conocimiento como base para la efectividad organizacional, la paulatina incertidumbre en los mercados y la globalización, entre otros (Bedoya, 2015). Este entorno ha consolidado un escenario competitivo, en el que las empresas deben dedicarse a fortalecer sus ventajas competitivas a través de la innovación y el sostenimiento de los activos de conocimiento para la supervivencia, la producción y el éxito de una empresa (Marulanda, López y López, 2016).

El término "gestión del conocimiento" fue utilizado por primera vez por Karl M. Wiig en 1986, quien lo definió como una sistemática construcción, renovación y aplicación explícita y deliberada de conocimiento para maximizar la efectividad relacionada con el conocimiento de una empresa. La gestión del conocimiento incorpora procesos organizacionales que buscan una combinación sinérgica de la capacidad de procesamiento de datos e información de las tecnologías de la información y la capacidad creativa e innovadora de los seres humanos (Voronov y Lavrinenko, 2014).

Durante mucho tiempo, las empresas querían llevar al nivel consciente lo que sabían cómo hacer, pero que hasta cierto punto no habían tenido tiempo de analizar; intentaron ir más allá de la comprensión de cómo pueden hacer el mejor uso de los conocimientos que poseen, lo que las ha llevado a desarrollar procedimientos sistemáticos para gestionarlo (Cepeda et al., 2017).

Es por esto que el propósito de esta revisión sistemática de la literatura se enfoca en identificar la intervención de la gestión del conocimiento en la competitividad de las organizaciones, y para lograrlo, se estudiaron los conceptos y puntos de vista sobre la gestión del conocimiento y la competitividad para posteriormente buscar su relación y aplicación a nivel organizacional. Seguido a ello, se describen los sectores de mayor representatividad y, por último, se presentan las dimensiones que son consideradas por los autores consultados. En consecuencia, es importante conocer algunas posturas de autores relacionados a la temática central del estudio: gestión del conocimiento y competitividad.

\section{Gestión del conocimiento}

Para Hernández y Calderón, citados por Sánchez (2016), la gestión del conocimiento se abre hacia dos campos de estudio: la economía de la innovación y la economía de las competencias; quiere decir, que el conocimiento es la capacidad de actuar, procesar e interpretar información para generar más conocimiento 0 dar solución a un determinado problema.

Contreras y Díaz (2014) definen la gestión del conocimiento en una organización, como la creación, transferencia, evaluación, disponibilidad y puesta en práctica del conocimiento. Además, retoman varias definiciones en las que se relaciona la gestión del conocimiento con la creación de valor para la compañía conducentes al logro de un negocio.

Sánchez (2016), cuando hace referencia a Chumjit (2012) y a Fallad (2011), afirma que la gestión del conocimiento es una integración de procesos dinámicos para obtener información y poder transformarla en conocimiento; afirma también, que la gestión del conocimiento trae consigo una formulación de políticas estratégicas que ayudan a desarrollar, mejorar y aplicar el conocimiento para el uso óptimo dentro de una organización con el fin de alcanzar sus objetivos y trae consigo muchos más beneficios cuantificables que se fundamentan en crecimiento y apoyo individual (Chalhoub, 2009). 
Según Porret (2010), citado por Contreras y Díaz (2014), la gestión del conocimiento es aquel enfoque orientado a encontrar y utilizar las mejores prácticas y procesos susceptibles de ser fuente de ventajas competitivas sostenibles, con el propósito de que los empleados puedan obtener en el momento adecuado la información que requieren para actuar con mayor eficiencia, idea que Tanascovici y Hagiu (2013) han denominado conocimiento de la organización.

Portillo (1998), mencionado por Castillo y D'Vicente (2009), define la gestión del conocimiento como el proceso que permite planificar, organizar, integrar y controlar el acceso y uso oportuno del conocimiento, el cual reside en el colectivo (conocimiento tácito) y en la infraestructura formacional (conocimiento explícito) de la organización para desarrollar activos del conocimiento, incremento de la efectividad y eficiencia, innovaciones en los productos y servicios, aumento de la productividad, y anticiparse a las tendencias y exigencias del entorno. Se identifica como una oportunidad competitiva para la consolidación de las pymes (Hernández, Marulanda y López, 2014).

También se puede definir la gestión del conocimiento como una forma de agregar o crear valor a través de la activación de los conocimientos, de la experiencia dentro y fuera de la organización y el uso de herramientas y técnicas específicas, de tal manera que el conocimiento está disponible cuando la organización necesita resolver problemas o encontrar soluciones (Meihami y Meihami, 2014). Los usos y razones del uso de la gestión del conocimiento son variados, partiendo desde generar cambios y resultados sustentables, optimizar recursos, aprovechar el conocimiento existente, aprender permanentemente 0 estimular la creatividad e innovación (Mracek y Mucha, 2013).

Para Sukmawati (2015) la gestión del conocimiento es toda actividad que utiliza el conocimiento para alcanzar el objetivo de la organización, de prepararla para superar el desafío del medio ambiente (entiéndase entorno) y mantener la competitividad en el mercado. Por otra parte, Tinoco, Salas y Tinoco (2014) retoman lo mencionado por Guerra (2008), quien afirma que la gestión del conocimiento vincula las necesidades de este con la estrategia de la empresa, dando acceso y representando tanto el saber individual como el organizativo, haciendo que como activo cree valor por sí mismo al ser aplicado en el proceso.

\section{Competitividad}

Si bien no es evidente el consenso acerca de la definición del concepto competitividad, sí se encuentra acuerdo en que debe ser entendida desde la empresa (enfoque micro), desde el sector económico (enfoque meso) y desde lo nacional o global (enfoque macro).

Desde la óptica macro, se encuentran tres enfoques: del Foro Económico Mundial (WEF), que la define como la habilidad que tienen los países para proveer altos niveles de prosperidad a sus ciudadanos; del Instituto Mexicano para la Competitividad (IMCO), para quien significa la habilidad de una región para atraer y retener inversiones; y la del Anuario de Competitividad Mundial, que afirma que la competitividad de las naciones analiza los hechos y políticas que determinan la capacidad de una nación para crear y mantener un entorno que sustente la generación de mayor valor para sus empresas y más prosperidad para su pueblo (Larios, 2016). Sin embargo, no necesariamente las empresas que más saben obtienen mejores resultados, sino más bien, las que logran usar mejor lo que saben (Andreu, 2016).

Desde el enfoque meso, la competitividad se define como la capacidad de un sector para mantener 0 aumentar, en condiciones de libre competencia, su participación en los mercados interior y exterior, a la vez que mantiene un crecimiento satisfactorio de los ingresos reales generados por su actividad (Bernal, Fracica y Frost, 2012). 
La competitividad desde el enfoque micro, es definida por Larios (2016) como la capacidad que tiene una organización pública o privada de mantener ventajas que le permitan alcanzar y mejorar la posición socioeconómica ante otras empresas. La competitividad define la capacidad de una empresa para producir bienes y servicios para mercados donde compite, manteniendo 0 incrementando su cuota de participación o nivel de rentabilidad (Bernal et al., 2012). La competitividad también incluye, a través de la producción y la aplicación del conocimiento, la capacidad de desarrollar procesos que faciliten la búsqueda de nuevas oportunidades y superar posibles obstáculos técnicos y organizativos (De Cuffa, Rojo y Maccari, 2015).

\section{METODOLOGÍA}

La metodología utilizada para identificar el aporte de la gestión del conocimiento a la competitividad de las organizaciones, fue una revisión sistemática de literatura a través de la cual se examina lo que se conoce hasta el momento sobre un tema determinado, cómo se ha investigado y las herramientas intelectuales y analíticas que podrían ayudar a su comprensión, como lo describe Ravitch (2016). Se caracteriza por partir de un propósito claro utilizando métodos sistemáticos y explícitos para localizar, seleccionar datos e información (Sánchez, 2010) y tiene como finalidad establecer relaciones, diferencias, etapas, posturas o estado actual del conocimiento respecto al fenómeno objeto de estudio. La revisión de la literatura ayuda a entender "la conversación" que tienen los expertos sobre un tema en particular (Ravitch, 2016).

Otro concepto sobre revisión de la literatura es el que plantea Locke (2010), quien afirma que consiste en la búsqueda de objetos de valor en los informes de investigación. Esos objetos son nuevos términos, palabras clave usadas en las búsquedas, referencias de otras publicaciones e investigadores, formas de redactar las preguntas de investigación, teorías novedosas, e identificación de indicadores de validez sobre los resultados de investigación.

Esta revisión se fundamentó en la exploración y análisis del objeto de estudio de 150 artículos publicados en revistas indexadas desde el 2007 y hasta el 2017, de los cuales 50 aportaban de manera directa al objetivo del fenómeno de estudio. El proceso de búsqueda se efectuó en bases de datos científicas como: CiteSeerX, Dialnet, DOAJ, EBSCO, Ebrary, Eumed.net, JSTOR, Scielo, Scipress y Redalyc. Se emplearon criterios de búsqueda tanto en español como en inglés: "gestión del conocimiento" o "competitividad". En esta etapa se realizó la lectura crítica de los 27 artículos seleccionados por su cercanía con la temática de estudio. De cada texto se identificaron elementos (sectores productivos) y dimensiones (respecto a la característica identificada en el sector productivo, como: innovación, estrategia, ventaja competitiva y tecnología, entre otras) que facilitaron la organización de la información explorada.

\section{RESULTADOS Y DISCUSIÓN}

La revisión realizada permite identificar algunos elementos (sectores) y dimensiones (características de los sectores) que reconocen la incursión de la gestión del conocimiento en la competitividad de las organizaciones, además de algunos sectores donde esta interrelación es significativa, como se presenta en la Figura 1.

Se confirma lo expuesto por Arellano (2015), que retoma los planteamientos de varios autores que dan relevancia a la relación de la gestión del conocimiento y la competitividad de las organizaciones, en esta época donde el conocimiento y la información son determinantes de la competitividad y la productividad. 
Bedoya (2015) retoma lo propuesto por Davenport (1999), quien sostiene que la gestión del conocimiento permite el logro de cuatro objetivos estratégicos: primero, crear almacenes de conocimiento, donde se recoge el conocimiento externo e interno; segundo, proporcionar acceso al conocimiento o facilitar su transferencia entre individuos; tercero, construir un entorno que permita la creación, transferencia y uso del conocimiento de manera más eficiente; y cuarto, lograr que la organización se concentre en el incremento de la utilización eficiente de los activos y en mejorar los resultados organizativos.

Figura 1. Relación de la gestión del conocimiento y competitividad en las organizaciones

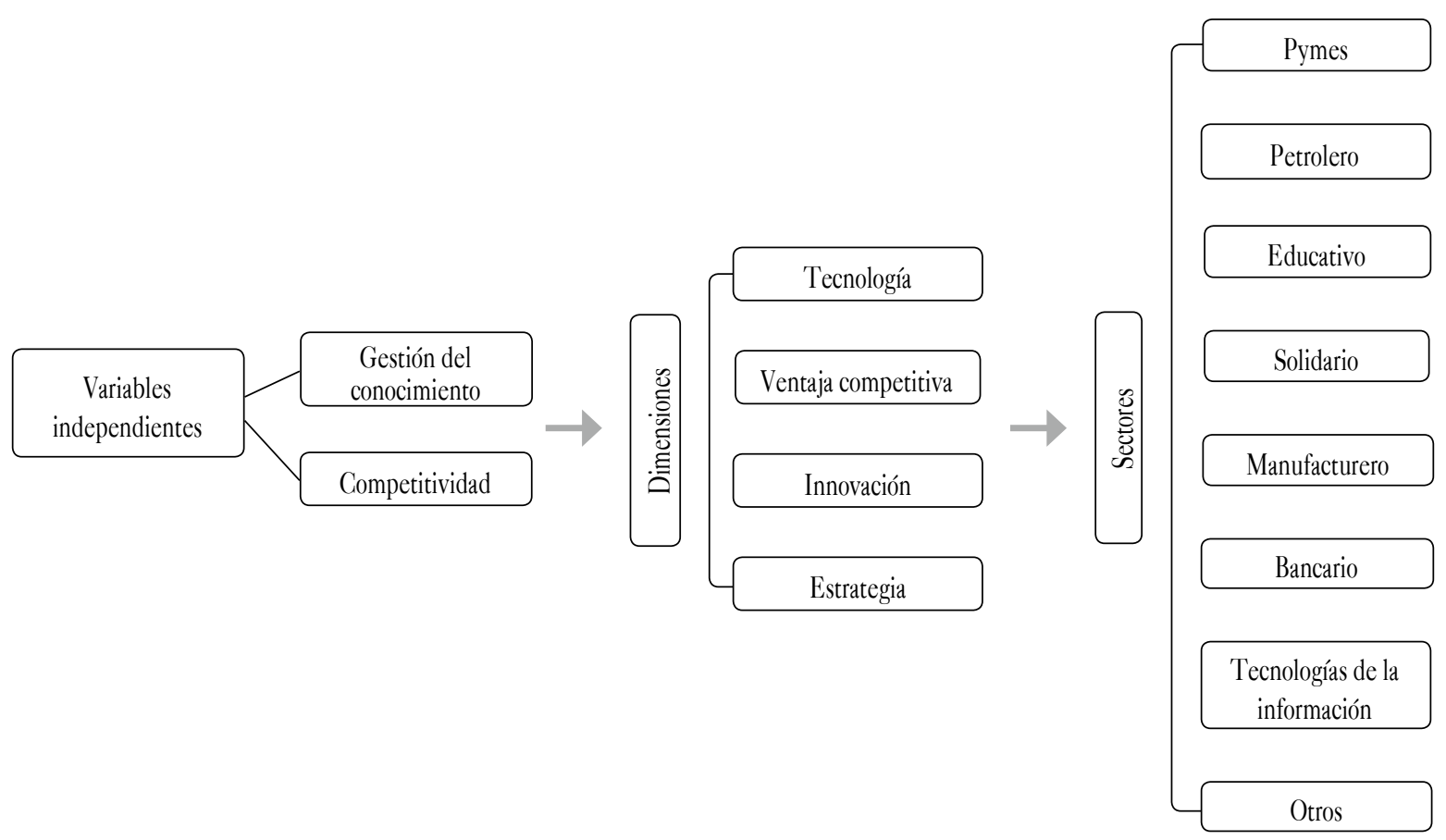

Fuente: elaboración propia.

Autores como Galvis y Sánchez (2014), permiten evidenciar la dualidad en la relación de la gestión del conocimiento y la competitividad, pues si bien la consideran como un elemento esencial para el mejoramiento, la innovación y la generación de ventajas competitivas sostenibles, también reconocen que la gestión del conocimiento se ha consolidado en forma de modelos que no brindan la claridad suficiente para llevarlos a la práctica en las organizaciones, razón por la cuál no sería tan claro su impacto en la competitividad (Sáiz, Manzanedo, del Olmo y Alcalde, 2009).
La exploración bibliográfica permitió la identificación de algunos sectores económicos (elementos) donde la gestión del conocimiento es reconocida como factor importante de la competitividad, y en este mismo sentido, se han determinado las dimensiones asociadas (características del sector) al objeto de estudio, los cuales se presentan en la Tabla 1.

Identificados los sectores, se hace fundamental entender que la información debe convertirse en conocimiento, y para que esto ocurra, son fundamentales 
las características de dichos sectores (dimensiones), como el liderazgo, la programación de estrategias, la organización de la estructura y la tecnología, entre otras. Lo anterior permite que se estructure la gestión del conocimiento y que esta se trasforme en gestión organizacional facilitando que el conocimiento entre como recurso a la organización y salga como resultado; que deje de ser privativo de un grupo exclusivo de especialistas y a una filosofía, donde el conocimiento útil es compartido y divulgado a toda la organización y reconoce la pérdida de conocimiento (no saber lo que se sabe) (Mayorga, Sánchez y González, 2016).

Tabla 1. Sectores económicos y dimensiones que relacionan los autores con respecto a la incursión de la gestión del conocimiento y la competitividad

\begin{tabular}{|c|c|c|}
\hline Sectores & Dimensiones & Autor \\
\hline \multirow{5}{*}{ Pymes } & Desempeño & (Vásquez et al., 2014) \\
\hline & Capital intelectual & (Gómez et al., 2014) \\
\hline & Cultura organizacional & (Larios, 2016) \\
\hline & Innovación & (Marulanda et al., 2013) \\
\hline & Estrategias & (Quintero, 2003) \\
\hline Petrolero & Tecnología & (Arellano, 2015) \\
\hline \multirow{8}{*}{ Educativo (educación superior) } & Tecnología & (Christer y Acevedo, 2015) \\
\hline & Innovación & (Vizcaíno, 2016) \\
\hline & \multirow{2}{*}{ Capital intelectual } & (Topete et al., 2012) \\
\hline & & (Díaz, 2007) \\
\hline & Investigación & (Castillo y D’Vicente, 2009) \\
\hline & Transferencia del conocimiento & (Chumjit, 2012) \\
\hline & Ventaja competitiva & (Águila, 2005) \\
\hline & Multidimensionalidad & (Clemenza et al., 2007) \\
\hline Solidario (tercer sector) & Innovación & (Bedoya, 2015) \\
\hline \multirow{2}{*}{ Manufacturero } & Innovación & (Peña et al., 2016) \\
\hline & Ventaja competitiva & (Meihami y Meihami, 2014) \\
\hline Bancario & Estrategia & (Sukmawati, 2015) \\
\hline \multirow{3}{*}{ Tecnologías de la información } & Innovación & (El-Kot y Gamal, 2011) \\
\hline & \multirow{2}{*}{ Ventaja competitiva } & (De Cuffa et al., 2015) \\
\hline & & (Majors, 2010) \\
\hline \multirow{6}{*}{ Sin clasificar } & Innovación & (Bernal et al., 2012) \\
\hline & Ventaja competitiva & (Montejano, 2016) \\
\hline & Tecnología & (Contreras y Díaz, 2014) \\
\hline & Innovación & (Silva et al., 2017) \\
\hline & Ventaja competitiva & (Rahimli, 2012) \\
\hline & Gestión de la calidad total & (Mihi Ramírez, 2008) \\
\hline
\end{tabular}

Fuente: elaboración propia. 
A nivel mundial, la gestión organizacional se encuentra representada por las pymes, agrupación de organizaciones con características de competitividad y productividad que aportan en promedio el $80 \%$ de la economía de los países. Es por esto que dentro de la revisión de la literatura se destaca su valor y aporte, como se describe a continuación. Seguidamente, se enfatizan los sectores productivos específicos en los que se está aplicando la gestión del conocimiento con un enfoque en la competitividad.

\section{Pymes}

Las pymes generalmente se enfrentan a una problemática común que tiene que ver con una inversión 0 implementación escasa o nula de sistemas de gestión del conocimiento dentro de las organizaciones y un desconocimiento de las ventajas que esta implementación podría generarles, además de una insuficiente introducción de tecnología que soporte el proceso. Lo anterior hace que estas organizaciones estén menos preparadas para enfrentar factores externos como la competencia desleal y los tratados de libre comercio. Las empresas de este tipo, a través del uso eficiente de los recursos productivos, desarrollan y aplican nuevas tecnologías para incrementar su competitividad (Vásquez, Guerrero y Núñez, 2014).

Los indicadores clave que inciden en la competitividad de las pymes son los externos, los que tienen que ver con el entorno de los negocios, la tecnología e innovación que se mide en la capacidad para lanzar nuevos productos al mercado, la calidad, la gestión de los recursos humanos, las capacidades directivas en el uso de nuevas herramientas de gestión, la internacionalización entendida como la capacidad para llegar a los mercados externos y el financiamiento como una fuente necesaria para su permanencia (Larios, 2016).

La gestión del conocimiento se considera fundamentalmente para los productos y procesos de innovación y mejora, para la toma de decisiones ejecutivas, y la adaptación y renovación de la organización (Marulanda, López y Mejía, 2013). Complementa Quintero (2003) afirmando que estas organizaciones desarrollan estrategias más integrales y sostenibles para ser competitivas frente a sus oponentes, y lo consolidan Gómez, Demuner y Gómez y Arriaga (2014) al considerar la importancia de integrar los ambientes internos y externos de la organización con la tecnología.

\section{Sector petrolero}

En las organizaciones petroleras se recomienda la aplicación del modelo de gestión del conocimiento PTCG (procesos, tecnologías, capital intelectual y cultura), el cual recomienda cuatro dimensiones principales que pueden generar ventajas competitivas; estas son: procesos de gestión, tecnología de la información y la comunicación, capital intelectual y cultura organizacional (Arellano, 2015).

Este último autor define la gestión del conocimiento como un conjunto de procesos y sistemas que permiten que el capital intelectual de una organización se incremente de forma significativa mediante la gestión de sus capacidades para la solución de problemas y cuyo objetivo es crear ventajas competitivas. Implementar un proceso de gestión del conocimiento implica que una organización ha identificado el capital intelectual que posee y lo ha incorporado a su proceso competitivo. Steward (1988), citado por Arellano (2015), define el capital intelectual como "el material intelectual, el conocimiento, la información, la propiedad intelectual y la experiencia que puede utilizarse para crear valor".

\section{Sector educativo (educación superior)}

La globalización ha cambiado todo nuestro mundo, la educación universitaria ha tenido que participar en la carrera por la subsistencia. Un factor de extrema importancia para mantenerse y ganar esta carrera estriba 
en ser competitivo, que según Águila (2005), "básicamente consiste en poseer calidad y que esta sea acreditada". De esta manera, puede decirse que la calidad de la educación es un concepto dinámico, que cambia con el tiempo, es diverso, pues varía según el contexto social, es multidimensional, puesto que es producto de diversas condiciones, y es total, dado que implica una atención en las diferentes dimensiones del aprendizaje (Clemenza, Ferrer y Pelekais, 2005).

Tanto la calidad como la competitividad dependen del capital intelectual de las personas (Topete, Bustos y Bustillo, 2012; Díaz, 2007): las actitudes ante los retos, habilidades, capacidades de innovar, intuición, creatividad, saber escuchar y comunicase con otros, hallar y usar información, plantear y resolver problemas, trabajar en equipo e individualmente, capacidad de aprender, tener responsabilidad, tenacidad, poseer valores y sensibilidad social (Castillo y D'Vicente, 2009). Según Vizcaíno (2016), las instituciones de educación superior que realicen gestión del conocimiento con mayores recursos tecnológicos lograrán mayor competitividad.

La gestión del conocimiento en el campo de la educación incentiva la formación, el intercambio y la transmisión de conocimientos a través de la tecnología, como instrumento que se utiliza para facilitar y desarrollar el conocimiento en forma amplia y cuyo manejo permite la transferencia y utilización del conocimiento, con lo que se mejora la estructura organizacional, la innovación y el aprendizaje de las instituciones (Chumjit, 2012).

Es importante identificar la comunicación y las tecnologías de la información como parte esencial de la gestión del conocimiento. La primera, que permite compartir, capturar y almacenar el conocimiento de la organización, y la segunda, puesto que ha creado una infraestructura para dirigir el conocimiento y dinamizar las oportunidades de gestión del mismo. Sin embargo, no es suficiente invertir en tecnología si se descuidan o subestiman las cuestiones culturales en el ambiente organizacional, pues estas juegan un papel importante en determinar el éxito general de las iniciativas de gestión del conocimiento (Christer y Acevedo, 2015).

\section{Sector solidario (tercer sector)}

En este sector, la gestión de conocimiento representa un conjunto de conceptos, métodos y tecnologías que permiten a los miembros de una organización trabajar juntos en una dirección definida por la empresa y establecer un vínculo entre la información disponible, la creación del conocimiento y el desarrollo de las competencias individuales y colectivas, siendo el concepto cooperativo la fundamentación de la economía solidaria. De esta forma, el conocimiento complejo, específico y tácito genera para las organizaciones ventajas competitivas duraderas porque es difícil de imitar, para generar una diferenciación o un conjunto de procesos sistemáticos entre los que se encuentran la identificación y captación del capital intelectual, el desarrollo y el compartimiento del conocimiento, orientados al desarrollo organizacional (Bedoya, 2015).

\section{Sector manufacturero}

Un estudio realizado en este sector por Peña Vega y Castellanos (2016), indica una directa relación entre la innovación, la gestión del conocimiento y la competitividad, pues las empresas manufactureras muestran evidencias a partir del aumento de las ventas de nuevos productos, la ampliación de la cuota de mercado, la diversificación del portafolio, acceso a nuevos mercados y la disminución de costos. Estas empresas utilizaron diferentes estrategias para gestionar el conocimiento, ya sea mediante la generación de conocimiento al interior de esta o mediante la transferencia de conocimientos desde el exterior hacia el interior de la organización. Así mismo, se identificaron prácticas para la distribución del conocimiento al interior de las compañías y para mejorar su uso. 
Es importante tener en cuenta que la gestión del conocimiento, en el sector manufacturero, se ha centrado en capturar el conocimiento acumulado en las mentes de los empleados individuales, para recopilarlo, gestionarlo y compartirlo con el resto de la organización, mejorando el desempeño organizacional. Uno de sus objetivos es mejorar la gestión de los procesos de conocimiento interno para que toda la información requerida para las decisiones corporativas pueda estar disponible y sea utilizada de manera eficiente (Meihami y Meihami, 2014).

\section{Sector bancario}

Sukmawati (2015), en su estudio, indica que las iniciativas de gestión del conocimiento pueden aplicarse a toda la organización o solo a un departamento o unidad; sin embargo, es necesario que exista una alineación entre la gestión del conocimiento y la estrategia empresarial. Las organizaciones, y en particular, las del sector financiero, pueden optar por dos tipos de estrategias según su necesidad: si la empresa necesita ser más eficiente en el proceso y debe reutilizar información se sugiere la estrategia de codificación, mientras que para organizaciones que necesitan innovación de productos, servicios o procesos, es más útil la estrategia de personalización.

El objetivo de la estrategia de codificación es recopilar conocimiento, guardarlo en una base de datos y presentarlo en forma explícita y codificada. El uso de conocimiento explícito puede ahorrar tiempo y dinero. El diseño de la base de datos, la gestión de documentos y la gestión del flujo de trabajo se consideran parte de la estrategia. El enfoque de la estrategia de personalización no es guardar el conocimiento, sino posibilitar a las personas comunicarlo; su objetivo es transferir, comunicar y mejorar el conocimiento a través de una red de conocimiento, como un foro de discusión (Sukmawati, 2015).

\section{Sector tecnologías de la información (TI)}

Según De Cuffa et al. (2015), para las empresas de este sector los beneficios de la gestión del conocimiento son claros, ya que a través de este activo pueden tener una mayor capacidad para compartir información a fin de facilitar el proceso de innovación y la competitividad del mercado. Para El-Kot y Gamal (2011), la gestión del conocimiento afectará positivamente la capacidad de innovación de estas organizaciones, la hace factible y permite la mejora continua y sostenida de los productos.

Takeuchi y Nonaka (2008), mencionados por De Cuffa et al. (2015), hacen referencia al conocimiento como un activo creado solo por individuos de tal manera que una organización no puede crear conocimiento sin la presencia de un elemento humano. Por lo tanto, la producción de conocimiento ocurre por interacción entre lo tácito y lo explícito. Las personas generan el primer tipo de conocimiento específico de un contexto particular y está relacionado con las percepciones (modelos mentales) de cada individuo y que es difícil de expresar, codificar y transferir, puesto que se convierte en un "ecosistema" (Majors, 2010). Mientras que el segundo tipo de conocimiento se refiere a uno más fácil de transmitir en lenguaje formal y sistemático a través de técnicas, métodos y estándares.

De Cuffa et al. (2015), retoman a Batista (2004) y Davenport y Prusak (2003), quienes afirman que debe tenerse en cuenta que la institucionalización ocurre de manera más efectiva cuando es respaldada por TI y comunicada de forma efectiva, de modo que el personal se sienta motivado para contribuir al éxito de este proceso. En el momento en que las empresas utilizan las prácticas similares, estandarizadas y formalizadas, el intercambio ocurre más rápidamente y su crecimiento se vuelve sistemático y colectivo, aparte del hecho de que el capital humano se vuelve más productivo. 


\section{Sectores sin clasificar}

Dentro de la exploración bibliográfica, algunos autores no hacen referencia a un sector productivo específico; sin embargo, están directamente relacionados con el objeto de estudio de esta revisión y están alineados en algunos casos a las dimensiones identificadas.

La organización que quiere realizar la implementación de un modelo de gestión del conocimiento debe generar un ambiente adecuado, además de tener una infraestructura y tecnología acordes a las funciones organizacionales, dando énfasis en los activos materiales 0 tangibles que permitan una permeabilidad hacia los activos intangibles de la organización ya que el conocimiento es demasiado abstracto para gestionarse, este se encuentra inmerso en la mente de las personas en forma de experiencias, conocimiento y valores (Contreras y Díaz, 2014).

Nonaka y Takeuchi (1998) y Saint-Onge (1999), mencionados por Bernal et al. (2012), mencionan que el conocimiento se entiende como un activo que tiene la capacidad de generar valor y producir riqueza, por lo cual requiere ser protegido para preservar la fuente de la ventaja competitiva de la empresa. Las organizaciones adquieren una ventaja competitiva sostenible a través de la adquisición y desarrollo del conocimiento organizacional y el fortalecimiento del capital humano, por lo tanto, no debería ser fácil para los competidores imitarla (Rahimli, 2012).

Para que este conocimiento se mantenga como fuente de ventaja competitiva, se requiere necesariamente de la tecnología de la información y la comunicación para administrarlo y optimizar su apropiación, generación y uso, además del establecimiento de redes y asociaciones entre empresas (Silva, Leal, Marques y Ferreira, 2017). De esta manera, se estará formalizando la innovación como estrategia organizacional; sin embargo, en algunas organizaciones no se identifica una relación directa entre la gestión del conocimiento y la innovación. Lo anterior podría ser causado porque no identifican la innovación, como la definirían Sedziuviene y Vveinhardt (2010), como la capacidad de aprendizaje, disponibilidad continua para reorganizarse, capacidad para iniciar cambios, crea un valor agregado en los mercados, ser creativos y poseer libertad de pensamiento.

En este punto también es importante considerar el estudio realizado por Montejano (2016) con $149 \mathrm{em}$ presarios en Aguascalientes (México), y que dio como resultado una dependencia directa entre ventajas competitivas y la disponibilidad de capital intelectual en las empresas.

Mihi Ramírez (2008) relaciona la gestión del conocimiento y la calidad total y encuentra cinco grandes campos de gran relevancia en esta relación: el liderazgo y compromiso de la dirección; la gestión de recursos humanos; la relación con clientes y proveedores; la cultura interna de la organización, y la gestión de los procesos. Observamos que dichos campos también son tratados con la gestión del conocimiento, la gestión de la calidad en ellos impulsa la gestión del conocimiento en la organización.

\section{CONCLUSIONES}

Dentro de la revisión realizada se identifica que el $18.5 \%$ de los artículos consultados hacen referencia a la gestión del conocimiento en el sector de las pymes; sin embargo, no es posible identificar sistemas formales de gestión del conocimiento que permitan articular todas las iniciativas y fortalecer estrategias de competitividad, productividad e innovación. Una forma de aportar en la mejora de esta situación, como lo sugieren Marulanda y López (2013), es avanzar en el uso y la apropiación de TIC de nueva generación, como web social y web semántica, y herramientas para el 
trabajo colaborativo, entre otras. Estas tecnologías digitales permiten gestionar información y conocimiento potenciando lo local y lo regional en un marco global.

El 22.2\% de los artículos revisados están relacionados con estudios generales que no se concentran en un sector productivo específico, y el $29.7 \%$ se encuentran en el sector educativo, destacando el gran aporte de los procesos de aprendizaje para la competitividad organizacional.

Tabla 2. Representación de los sectores identificados

\begin{tabular}{|l|c|c|}
\hline Sectores & $\begin{array}{c}\text { N. }{ }^{\text {de }} \\
\text { artículos }\end{array}$ & $\begin{array}{c}\text { Porcentaje } \\
(\%)\end{array}$ \\
\hline Pymes & 5 & 18.5 \\
\hline Petrolero & 1 & 3.7 \\
\hline Educativo (educación superior) & 8 & 29.7 \\
\hline Solidario (tercer sector) & 1 & 3.7 \\
\hline Manufacturero & 2 & 7.4 \\
\hline Bancario & 1 & 3.7 \\
\hline Tecnologías de la información & 3 & 11.1 \\
\hline Sin clasificar & 6 & 22.2 \\
\hline
\end{tabular}

Fuente: elaboración propia.

En cuanto a las dimensiones identificadas, el $26 \%$ de los artículos revisados hace referencia a la innovación y el $11.1 \%$ a la tecnología (incluye infraestructura tecnológica y las tecnologías de la información y la comunicación), haciendo evidente el aporte de estas en la sistematización, disponibilidad y comunicación del conocimiento y como mediadoras de la gestión del conocimiento organizacional. El 37 \% corresponde a otras dimensiones sin clasificar y permite inferir que la competitividad a partir de la gestión del conocimiento puede verse intervenida por la gestión, ya sea por sistemas, iniciativas, prácticas o indicadores que fortalecen la administración de las organizaciones.

Se concluye que las TI favorecen el desarrollo de sistemas más descentralizados y estructuras flexibles que facilitan los procesos de generación y transformación de conocimiento, permitiendo que la información se difunda a lo largo de toda la organización, ayudando a descentralizar en la toma de decisiones, asegurando el compromiso de los empleados, estimulando el intercambio de información y fomentando el desarrollo del trabajo en equipo, comprobando lo expuesto por Pérez y Junquera (2013).

Por el contrario, dimensiones como la estrategia representan el $7.4 \%$ de los artículos revisados, lo cual sorprende si tenemos en cuenta lo propuesto por Shujahat et al. (2017) quienes relacionan la estrategia con los procesos de gestión del conocimiento y la innovación a través de la productividad del trabajador del conocimiento. En consecuencia, las organizaciones deben valorar a los trabajadores del conocimiento ya que son ellos los que utilizan las TI, la infraestructura tecnológica y otros elementos de gestión del conocimiento para mejorar su productividad y, por lo tanto, la innovación.

Otras dimensiones con baja presencia o nula en la revisión son el liderazgo y la cultura organizacional, situación que motiva una búsqueda específica del impacto de estas, puesto que estudios como los realizados por Jasimuddin y Naqshbandi (2018), Osorno, Oquendo, Monsalve y Martínez (2016) y Madhoushi y Sadati (2010), analizan su impacto e identifican que estas dimensiones tiene un efecto estadísticamente significativo en las capacidades de gestión del conocimiento, la innovación y la eficacia de las organizaciones. La relación de dimensiones identificadas se presenta en la Tabla 3.

Se logra establecer que el conocimiento se constituye en un principio fundamental de la ventaja competitiva de las organizaciones, como lo afirma Rahimli (2012), quien coincide con la mayoría de los artículos analizados puesto que concluye que la relación entre la gestión del conocimiento y la ventaja competitiva 
sostenible es altamente positiva a través del estudio que desarrolló.

Tabla 3. Representación de las dimensiones identificadas

\begin{tabular}{|l|c|c|}
\hline Dimensiones & $\mathbf{N}^{\circ}$ de artículos & Porcentaje (\%) \\
\hline Otros & 10 & 37 \\
\hline Innovación & 7 & 26 \\
\hline Ventaja competitiva & 5 & 18.5 \\
\hline Tecnología & 3 & 11.1 \\
\hline Estrategia & 2 & 7,4 \\
\hline
\end{tabular}

Fuente: elaboración propia.

Con la presente revisión sistemática de la literatura se genera una línea de interés a desarrollar, puesto que existen otras dimensiones que pueden tener una incidencia en la ventaja competitiva organizacional a partir de la gestión del conocimiento, como son: el liderazgo (Sánchez, 2008), la comunicación, la cultura organizacional y la gestión del recurso humano, entre otras, temáticas que no fueron relevantes en la búsqueda realizada pero sí de importancia en el desempeño organizacional.

En concreto, la competitividad de las micro, pequeñas y medianas empresas deberá entenderse como la capacidad de producir, ofrecer y comercializar productos o servicios innovadores y de alto valor agregado, en las condiciones, cantidad y calidad que el mercado del país y el del extranjero lo exigen, como lo expresa Larios (2016).

Además, con los resultados obtenidos se impulsan estudios relacionados con los sistemas de gestión del conocimiento y su relación con los sistemas de gestión y organización para fortalecer las ventajas competitivas de las organizaciones. También podría ser interesante profundizar en la medición de la relación de la gestión del conocimiento y la competitividad a través de indicadores como: rentabilidad relativa, nivel de costo, participación de mercado, generación de nuevos productos o servicios, tiempos de producción y lealtad de los compradores.

\section{REFERENCIAS}

Águila, V. (2005). El concepto calidad en la educación universitaria: clave para el logro de la competitividad institucional. Revista Iberoamericana de Educación, 36(12), 1-7. Recuperado de file://C:/Users/ Usuario/Downloads/2886-Texto\%20del\%20 art\%C3\%ADculo-832-1-10-20180118.pdf

Andreu, R. (2016). Gestión del conocimiento y competitividad. Navarra, España: Ediciones Universidad de Navarra, S. A.

Arellano, M. (2015). Gestión del conocimiento como estrategia para lograr ventajas competitivas en las organizaciones petroleras. Revista Cientifica Ciencias Humanas, 30(10), 31-47. Recuperado de http://www.redalyc.org/pdf/709/70932870004.pdf.

Bedoya Dorado, C. (2015). Gestión del conocimiento en el tercer sector: de la competitividad a la eficiencia organizacional. Entramado, 11(2), 94-111. Recuperado de http://revistasojs.unilibrecali.edu. co/index.php/entramado/article/viewFile/619/786

Bernal Torres, C. A., Fracica Naranjo, G., y Frost González, J. S. (2012). Análisis de la relación entre la innovación y la gestión del conocimiento con la competitividad empresarial en una muestra de empresas en la ciudad de Bogotá. Estudios Gerenciales: Journal of Management and Economics for Iberoamerica, 28, 303-315. Recuperado de file:///C:/Users/Usuario/Downloads/Dialnet-AnalisisDeLaRelacionEntreLaInnovacionYLaGestionDel-5444934.pdf 
Castillo, M., y D’Vicente, Y. (2009). Gestión del conocimiento y competitividad en el área de investigación y producción de los colegios e institutos universitarios privados de Maracaibo, Venezuela. Clío América, 3(6), 165-182. Recuperado de file://C:/Users/Usuario/Downloads/389-1-918-1-10-20140131.pdf

Cepeda-Carrión, I., Martelo-Landróguez, S., LealRodríguez, A. L., y Leal-Millán, A. (2017). Critical processes of knowledge management: An approach toward the creation of customer value. European Research on Management and Business Economics, 23(1), 1-7. Recuperado de https:// ac.els-cdn.com/S2444883416300092/1-s2.0S2444883416300092-main.pdf?_tid=312c1111f92c-4b53-adcd-79a2bdbc7310\&acdnat $=1531097$ 227_1eb91c97209e6699c5485bfda44687c0

Chalhoub, M. (2009). Employee growth and development through knowledge management in the global environment: effects on the competitiveness of firms in a multinational context. Journal of Knowledge Globalisation, 2(2).

Christer Olsson, J. O., y Acevedo Tabares, J. C. (2015). El desarrollo de capacidades y la gestión del conocimiento en los potenciales de cambio y competitividad universitaria. Anagramas Rumbos y Sentidos de la Comunicación, 14(27), 201-215. Recuperado de http://www.redalyc.org/pdf/4915/491548261011.pdf

Clemenza, C., Ferrer, J., y Pelekais, C. (2005). La calidad como elemento competitivo en las universidades. Revista de Artes y Humanidades UNICA, 6(14), 55-86. Recuperado de file:///C:/ Users/Usuario/Downloads/art\%C3\%ADculo_redalyc_170118766004.pdf

Contreras-Medina, D. I., y Díaz-Nieto, E. S. (2014). La gestión del conocimiento factor clave de competitividad. Un estudio de los modelos y paradigmas. Contribuciones a la Economía, (Abril 2014). Recuperado de http://www.eumed.net/ce/2014/2/ conocimiento-competitividad.html

Chumjit, S. (2012). Knowledge management in higher education in Thailand (tesis de doctorado). The University of Texas at Arlintong, Texas, EE. UU.

De Cuffa, D., Rojo, C. A., y Maccari, E. A. (2015). Knowledge management in a competitive scenario of an information technology company. International Journal of Innovation, 3(1), 17-34. Recuperado de http://www.journaliji.org/index.php/iji/ article/viewFile/69/pdf_24

Díaz, L. V. (2007). Gestión del conocimiento y del capital intelectual: una forma de migrar hacia empresas innovadoras, productivas y competitivas. Revista Escuela de Administración de Negocios, (61), 39-68. Recuperado de https://journal.universidadean.edu.co/index.php/Revista/article/ viewFile/415/409

El-Kot, G., y Gamal, D. (2011). How does knowledge management drive competitiveness in Egyptian software companies? Review of Management, 1(4), 56-76. Recuperado de http://papers.aast.edu/ staffpdf/24575_7_79_Review\%20of\%20Management, \%20Vol.\%201,\%20No.\%204,\%20December\%202011.pdf\#page=56

Galvis Lista, E., y Sánchez Torres, M. (2014). Revisión sistemática de literatura sobre procesos de gestión de conocimiento. Revista Tecnología Informática, 13(37), 45-67. Recuperado de file:///C:/Users/ Usuario/Downloads/4691-Texto\%20del\%20art\% C3\%ADculo-21584-1-10-20150320.pdf

Gómez Díaz, M. R., Demuner Flores, M. R., Gómez Díaz, A. E., y Arriaga Tapia, R. (2014). Gestión del conocimiento en pymes, una exploración. 
Vestigium Ire, 6(1), 19-25. Recuperado de file:///C:/ Users/Usuario/Downloads/785-2356-1-PB.pdf

Hernández, A., Marulanda, C. E., y López, M. (2014). Análisis de capacidades de gestión del conocimiento para la competitividad de pymes en Colombia. Información tecnológica, 25(2), 111-122.

Jasimuddin, S. M., y Naqshbandi, M. M. (2018). Knowledge-oriented leadership and open innovation: Role of knowledge management capability in France-based multinationals. International Business Review, 27(3), 701-713. Recuperado de file://C:/Users/Usuario/Downloads/2018IBR.pdf

Larios Gómez, E. (2016). La gestión de la competitividad en la mipyme mexicana: diagnóstico empírico desde la gestión del conocimiento. Revista de Administração da Unimep, 14(2), 177209. Recuperado de http://www.redalyc.org/ pdf/2737/273746863008.pdf

Locke, L. (2010). Reading and Understanding Research. Washington D. C.: SAGE Publications.

Madhoushi, M., y Sadati, A. (2010). Knowledge management, antecedent of organizational innovation and competitiveness. In Proceedings of the European Conference on Intellectual Capital (pp. 391-398). Recuperado de https://www. researchgate.net/profile/Neagu_Ana_Maria/ publication/284593746_Knowledge_Dynamics_the_Learning_Cycle_and_Intellectual_ Capital_A_Basic_Framework_for_Knowledge_ Internalization/links/56554de908aeafc2aabc46b4/ Knowledge-Dynamics-the-Learning-Cycle-andIntellectual-Capital-A-Basic-Framework-forKnowledge-Internalization.pdf\#page $=411$

Majors, I. (2010). ICT and knowledge management models for promotion of SME's competitiveness.
The International Journal of Technology, Knowledge and Society, 6(3), 173-184.

Marulanda Echeverry, C. E., López Trujillo, M., y Mejía Giraldo, M. (2013). Análisis de la gestión del conocimiento en pymes de Colombia. Gerencia Tecnológica Informática, 12(33), 33-44. Recuperado de file://C:/Users/Usuario/Downloads/ Dialnet-AnalisisDeLaGestionDelConocimientoEnPYMESDeColombi-4546794.pdf

Marulanda Echeverry, C. E., y López Trujillo, M. (2013). La gestión del conocimiento en las pymes de Colombia. Revista Virtual Universidad Católica del Norte, 1(38), 158-170. Recuperado de file://C:/ Users/Usuario/Downloads/411-2034-1-PB.pdf

Marulanda, C., López, M., y López, F. (2016). La cultura organizacional y las competencias para la gestión del conocimiento en las pequeñas y medianas empresas (pymes) de Colombia. Información tecnológica, 27(6), 03-10.

Mayorga Salamanca, P. I., Sánchez Gutiérrez, J., y González Uribe, E. G. (2016). Efectos de las estrategias de la gestión del conocimiento en la competitividad de las pymes. Red Internacional de Investigadores en Competitividad, 9(1), 17281749. Recuperado de file://C:/Users/Usuario/ Downloads/96-2373-1-PB.pdf

Meihami, B., y Meihami, H. (2014). Knowledge Management a way to gain a competitive advantage in firms (evidence of manufacturing companies). International Letters of Social and Humanistic Sciences, 14(3), 80-91. Recuperado de file://C:/Users/ Usuario/Downloads/article_176912.pdf

Mihi Ramírez, A. (2008). Un análisis de la gestión de la calidad total y de la gestión del conocimiento como fuente de ventajas competitivas. Universidad \&̊ 
Empresa, 10(14), 163-177. Recuperado de file:///C:/ Users/Usuario/Downloads/1051-3819-1-PB.pdf

Montejano, S. (2016). La competitividad y su relación con la gestión del conocimiento y el mercado global. Guadalajara, México: Editorial Universidad de Guadalajara.

Mráček, P., y Mucha, M. (2013). Advergaming: Application of knowledge in the process of the competitive advantage improvement. Trends Economics and Management, 5(8), 139-147. Recuperado de https://trends.fbm.vutbr.cz/index.php/trends/ article/viewFile/125/111

Osorno Balbín, A., Oquendo Gómez, E. M., Monsalve Trujillo, I. I., y Martínez Gómez, J. (2016). Gestión del conocimiento, innovación para el crecimiento empresarial. Science of Human Action, 1(1), 104-116. Recuperado de file:///C:/Users/Usuario/ Downloads/1917-7560-6-PB.pdf

Peña Vargas, L. H., Vega Durán, C. A., y Castellanos Méndez, J. G. (2016). Innovación y gestión del conocimiento para el incremento de la productividad empresarial. Memorias, 14(26). 93-110. DOI: http:// dx.doi.org/10.16925/me.v14i26.1571. Recuperado de file:///C:/Users/Usuario/Downloads/1571-44141-PB.pdf

Pérez-López, S., y Junquera, B. (2013). The relation between IT competency and knowledge management processes and its mediators. Tourism \& Management Studies, 9(1), 109-115. Recuperado de https://tmstudies.net/index.php/ectms/article/ viewFile/555/951

Quintero Ramírez, Á. (2003). El aprendizaje en la empresa: la nueva ventaja competitiva. Educación y educadores, 6(1), 127-139. Recuperado de file:///C:/ Users/Usuario/Downloads/531-2247-1-PB.pdf
Rahimli, A. (2012). Knowledge management and competitive advantage. Information and Knowledge Management, 2(7), 37-43. Recuperado de https://s3.amazonaws.com/academia.edu.documents/30312949/Knowledge_Management_and_ Competitive_Advantage.pdf?AWSAccessKeyI $\mathrm{d}=$ AKIAIWOWYYGZ2Y53UL3A\&Expires=1531108722\&Signature=cXWCPkeWmuC1hLMA pQ64nHRgX78\%3D\&response-content-dispositi on $=$ inline $\% 3 \mathrm{~B} \% 20$ filename $\% 3$ DIISTE_November_Issue._High_Impact_Factor.pdf

Ravitch, S. (2016). How Conceptual Frameworks Guide Research. Washington D. C.: SAGE Publications.

Sáiz, L., Manzanedo, M. Á., del Olmo, R., y Alcalde, R. (2009). La contribución de la gestión del conocimiento a la competitividad de la empresa. Su estudio en las pymes de Castilla y León. En XIII Congreso de Ingeniería de Organización (pp. 1730-1736). Barcelona-Terrassa, España. Recuperado de http://adingor.es/congresos/web/uploads/ cio/cio2009/1730-1736.pdf

Sánchez Manchola, I. D. (2008). Los estilos de dirección y liderazgo: propuesta de un modelo de caracterización y análisis. Pensamiento \& Gestión, (25), 1-39. Recuperado de http://www.scielo.org. co/pdf/pege/n25/n25a02.pdf

Sánchez Meca, J. (2010). Cómo realizar una revisión sistemática y un meta-análisis. Aula Abierta, 38(2), 53-64. Recuperado de file:///C:/Users/Usuario/ Downloads/Dialnet-ComoRealizarUnaRevisionSistematicaYUnMetaanalisis-3316651.pdf

Sánchez, J. (2016). La gesstión del conocimiento y la competitividad en las pymes. Guadalajara, México: Editorial Universidad de Guadalajara.

Sedziuviene, N., y Vveinhardt, J. (2010). Competitiveness and innovations: role of knowledge 
management at a knowledge organization. Engineering Economics, 21(5). 525-536. Recuperado de http://www.inzeko.ktu.tt/index.php/EE/article/ viewFile/11716/6385

Shujahat, M., Sousa, M. J., Hussain, S., Nawaz, F., Wang, M., y Umer, M. (2017). Translating the impact of knowledge management processes into knowledge-based innovation: The neglected and mediating role of knowledge-worker productivity. Journal of Business Research.

Silva, R., Leal, C., Marques, C. S., y Ferreira, J. (2017, March). The Strategic Knowledge Management, Innovation and Competitiveness: A Bibliometric Analysis. In ECIC 2017-9th European Conference on Intellectual Capital (pp. 303-311). Academic Conferences and publishing limited.

Sukmawati, S. (2015). A knowledge management strategy to achieve organisation competitiveness. International Journal of Scientific \& Technology Research, 4(8), 334-339.

Tanascovici, M., y Hagiu, A. (2013). Competitive intelligence in the knowledge-based organisation. Network Intelligence Studies, 1, 44-53. Recuperado de http://seaopenresearch.eu/Journals/articles/NI S_1_5.pdf

Tinoco Gómez, O. R., Salas Bacalla, J. A., y Tinoco Ángeles, F. E. (2014). Gestión del conocimiento y su incidencia en la competitividad empresarial de las mypes del sector textil y de confecciones en Lima Metropolitana. Theorèma - UNMSM, 1(1), 161-170. Recuperado de file://C:/Users/Usuario/ Downloads/11949-41663-1-PB.pdf

Topete Barrera, C., Bustos Farías, E., y Bustillos Ramos, E. S. (2012). Gestión del conocimiento para promover la productividad académica de los institutos tecnológicos en la sociedad del conocimiento. Sinéctica, (38), 1-15. Recuperado de http:// www.scielo.org.mx/pdf/sine/n38/n38a5.pdf

Vásquez Ávila, G., Guerrero Herrera, J. F., y Núñez Moreno, T. E. (2014). Gestión de conocimiento, capital intelectual y competitividad en pymes manufactureras en México. Retos, 4(7), 29-43. Recuperado de file:///C:/Users/Usuario/ Downloads/98-Texto\%20del\%20art\%C3\%AD culo-187-1-10-20150605.pdf

Vizcaíno, A. (2016). La competitividad y su relación con la gestión del conocimiento y el mercado global. Guadalajara, México: Editorial Universidad de Guadalajara.

Voronov, V., y Lavrinenko, O. (2014). Knowledge management and the incresase of SME competitiveness: a case study. Baltic Region, 3(21), 22-32. Recuperado de https://cyberleninka.ru/article/n/ knowledge-management-and-the-incresase-ofsme-competitiveness-a-case-study 